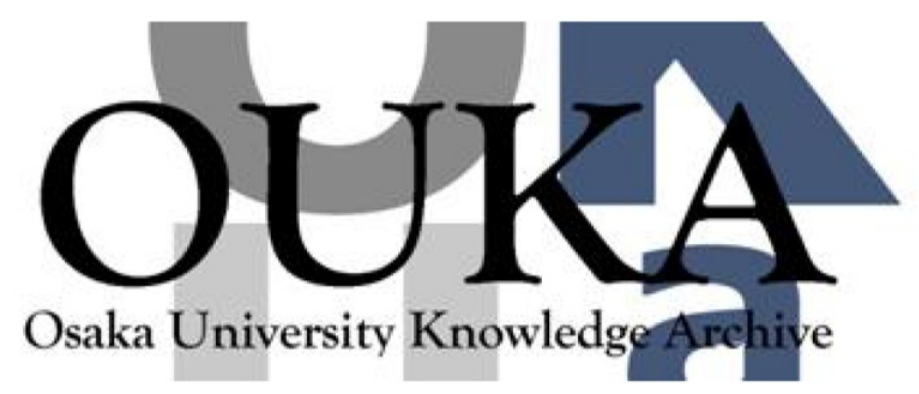

\begin{tabular}{|c|l|}
\hline Title & $\begin{array}{l}\text { Broad spectral sensitization of organic } \\
\text { photovoltaic heterojunction device by perylene } \\
\text { and C60 }\end{array}$ \\
\hline Author(s) & Feng, Wei; Fujii, A.; Lee, S. et al. \\
\hline Citation & $\begin{array}{l}\text { Journal of Applied Physics. 88(12) p. 7120- } \\
\text { p. 7120 }\end{array}$ \\
\hline Issue Date & $2000-11-28$ \\
\hline oaire:version & VoR \\
\hline URL & https://hdl. handle. net/11094/75667 \\
\hline rights & \\
\hline Note & \\
\hline
\end{tabular}

Osaka University Knowledge Archive : OUKA

https://ir. Library. osaka-u. ac. jp/

Osaka University 


\section{Broad spectral sensitization of organic photovoltaic heterojunction device by perylene and $\mathrm{C}_{60}$}

Cite as: Journal of Applied Physics 88, 7120 (2000); https://doi.org/10.1063/1.1323514

Submitted: 26 June 2000 . Accepted: 15 September 2000 . Published Online: 28 November 2000

W. Feng, A. Fujii, S. Lee, H. Wu, and K. Yoshino

\section{ARTICLES YOU MAY BE INTERESTED IN}

$2.5 \%$ efficient organic plastic solar cells

Applied Physics Letters 78, 841 (2001); https://doi.org/10.1063/1.1345834

$4.2 \%$ efficient organic photovoltaic cells with low series resistances

Applied Physics Letters 84, 3013 (2004); https://doi.org/10.1063/1.1713036

Small molecular weight organic thin-film photodetectors and solar cells

Journal of Applied Physics 93, 3693 (2003); https://doi.org/10.1063/1.1534621

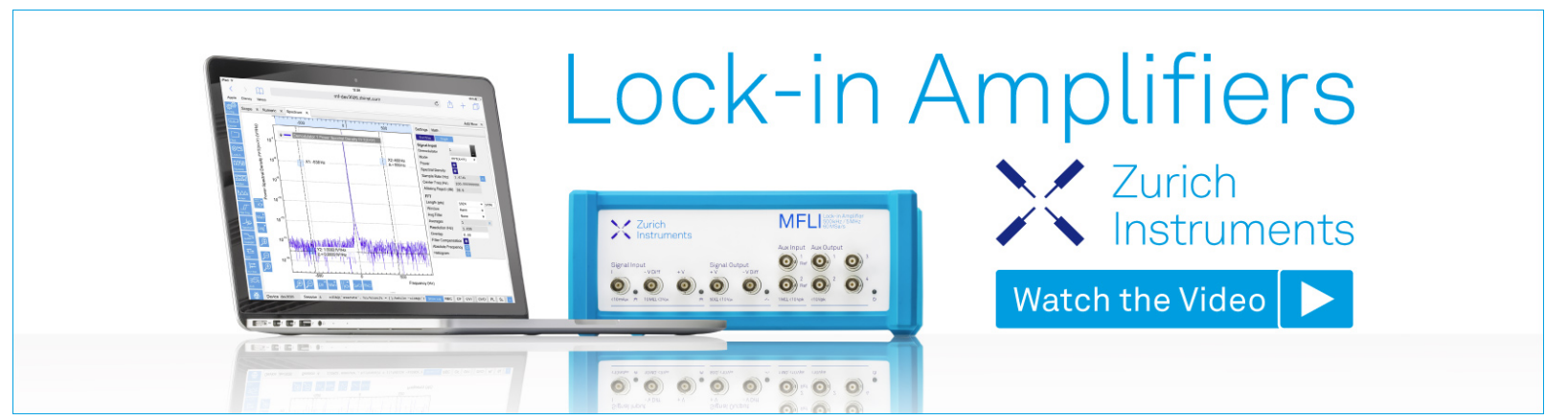




\title{
Broad spectral sensitization of organic photovoltaic heterojunction device by perylene and $\mathrm{C}_{60}$
}

\author{
W. Feng \\ Department of Electronic Engineering, Graduate School of Engineering, Osaka University, \\ 2-1 Yamada-Oka, Suita, Osaka 565-0871, Japan and School of Electronic and Information Engineering, \\ $X i$ 'an Jiaotong University, Xi'an, 710049, People's Republic of China \\ A. Fujii and S. Lee \\ Department of Electronic Engineering, Graduate School of Engineering, Osaka University, \\ 2-1 Yamada-Oka, Suita, Osaka 565-0871, Japan \\ H. Wu \\ School of Electronic and Information Engineering, Xi'an Jiaotong University, Xi'an, 710049, \\ People's Republic of China \\ K. Yoshino \\ Department of Electronic Engineering, Graduate School of Engineering, Osaka University, \\ 2-1 Yamada-Oka, Suita, Osaka 565-0871, Japan
}

(Received 26 June 2000; accepted for publication 15 September 2000)

\begin{abstract}
The characteristics of heterojunction devices consisting of indium-tin-oxide (ITO)/poly(3-alkylthiophene) (PAT)/ $N, N^{\prime}$-diphenyl glyoxaline-3, 4, 9, 10-perylene tetracarboxylic acid diacidamide (PV)/Al, ITO/PAT-PV/PV/A1, and ITO/PAT-PV-C 60 /PV/Al, which are typical photoinduced rectifiers, are reported. The device under dark conditions is almost insulating; however, when exposed to light marked rectification and strong photoresponse over a broad spectral range covering near-infrared to visible and $\mathrm{UV}$ wavelengths are observed. The effect of PV and $\mathrm{C}_{60}$ doping as well as annealing on spectral photoresponse and photoelectric conversion was investigated. The results show that the efficient photoinduced electron transfer from PAT to PV and $\mathrm{C}_{60}$ occurs at the interface between the composite and junction. (C) 2000 American Institute of Physics. [S0021-8979(00)04624-7]
\end{abstract}

\section{INTRODUCTION}

Conducting polymers (CPs) with extended $\pi$-conjugation in their main chains have attracted much attention not only from a fundamental viewpoint but also for practical interest as materials suitable for electrical and optoelectronic devices such as photodiodes and solar cells. ${ }^{1-3}$ Organic materials are attractive because of their light weight and processability, and the ease of material design on the molecular level. However, a fundamental problem in the design of organic photovoltaic devices such as Schottky and heterojunction devices is the fact that the charge-separating region is usually a fraction of the light-harvesting region. Ideally, every absorbed photon should produce a chargecarrier pair, which is separated at an active interface by the local electrical field. In reality, the majority of photons absorbed outside this charge-separating region fail to contribute to the photovoltaic current, because the absorption length of the incident light markedly exceeds the width of the chargeseparating region. In order to solve this problem, an interesting alternative was developed which involved mixing an electron donor with an electron acceptor material, whereupon a large interfacial area on a scale similar to exciton diffusion is created. ${ }^{4}$

Among the various conducting polymers, poly(3alkylthiophene) (PAT) has attracted much attention as a soluble and even fusible polymer, because of its interesting and novel properties. ${ }^{5,6}$ The electronic and optical properties of PAT as a photoactive material have been demonstrated in photovoltaic heterojunction devices which exhibit relatively high efficiency. ${ }^{7}$ PAT is a member of the family of quasione-dimensional conducting polymers which have relatively broad $\pi$ and $\pi^{*}$ bands and which when doped have properties that span the full range from insulator to metal. ${ }^{8}$ Moreover, among many conjugated polymers the PAT chain is very flexible. When annealed, the PAT chain arrays repeatedly reach the ordered state.

$\mathrm{PV}$ is a promising candidate for an electron acceptor material in photovoltaic devices, ${ }^{9}$ because PV exhibits relatively high electron affinity of the large-band-gap materials, large visible extinction coefficients, photostability and lowcost fabrication. ${ }^{10,11}$ Buckminsterfullerene, $\mathrm{C}_{60}$, possesses higher electron mobility than a conjugating polymer, and a higher electron affinity and ionization energy. ${ }^{12}$ The efficient quenching of photoluminescence obtained by mixing conjugated polymers with $\mathrm{C}_{60}$ or perylene is evidence of photoinduced charge transfer, with the $\mathrm{C}_{60}$ and perylene acting as electron acceptors. Many studies on the photophysics of mixtures of conjugated polymers with fullerene or dye have been reported. ${ }^{13,14}$ The results of these studies clearly showed an ultrafast reversible metastable photoinduced electron transfer from conjugated polymers onto $\mathrm{C}_{60}$ or perylene in solid films.

We have shown that the photocurrent of $\operatorname{poly}(2,5-$ dialkoxy-p-phenylene vinylene) (ROPPV) photovoltaic cells is increased upon the introduction of a soluble perylene de- 


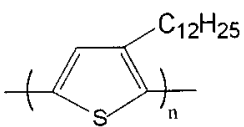

(a)

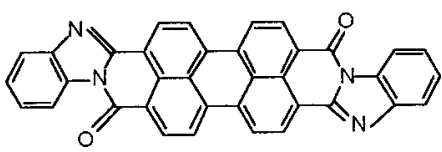

(b)
FIG. 1. Molecular structures of the poly(3-dodecylthiophene) (PAT12) (a) and perylene tetracarboxylic derivative (b).

rivative into the polymer. As well, upon annealing, such mixture films show a further enhancement of the absorption in the visible light region and of the crystallinity, ${ }^{15}$ which have been attributed to the formation of an electronconducting network of interconnecting perylene with polymer. The changes in morphological characteristics of composite films of conducting polymer-perylene have been studied and will be discussed elsewhere.

In this article, we report on the preparation of the PAT-PV-C 60 composite with a preselected ratio. The photovoltaic cells comprising the double-layer structure (ITO/PAT/PV/Al, ITO/PAT-PV/PV/Al and ITO/PAT-PV-C 60 PV/Al) were fabricated, and their (I/V) characteristics and photocurrent spectra are discussed.

\section{EXPERIMENT}

The poly(3-dodecylthiophene) (PAT12) was synthesized according to the method previously described. ${ }^{16}$ PAT12 is highly soluble in toluene and chloroform. $N, N^{\prime}$-diphenyl glyoxaline-3, 4, 9, 10-perylene tetracarboxylic acid diacidamide (PV) was synthesized according to the method previously described. ${ }^{15}$ The chemical structures of PAT-12 and PV are shown in Fig. 1. PAT12-PV-C 60 composites at appropriate weight ratios are dissolved in toluene while PV is dissolved in chloroform. The heterostructure is prepared by spin coating the PAT12-PV- $\mathrm{C}_{60}$ composite solution and the PV solution on a patterned ITO-coated glass substrate with a sheet resistance of $10 \Omega / \square$ followed by the deposition of an Al contact layer by thermal evaporation through a shadow mask. The active area of the device is $1 \times 1 \mathrm{~mm}^{2}$. The typical thickness of the first layer of all devices (PAT layer, PAT-PV layer and PAT-PV-C 60 layer for the single composition device, the double composition device and the triple composition device, respectively) formed by using spin coating is about $0.1-0.2 \mu \mathrm{m}$, and that of the second layer (PV layer) formed by using casting is about $10 \mu \mathrm{m}$. All samples and devices were measured before and after annealing at $150{ }^{\circ} \mathrm{C}$ for $1 \mathrm{~h}$.

The absorption and photoluminescence spectra were measured using a Hitachi $330 \mathrm{UV}$-vis spectrophotometer and fluorescence spectrophotometer (F-4500, Hitachi). Photoelectrical measurements were carried out in a vacuum optical cryostat under a vacuum of about $10^{-5}$ Torr. $I-V$ characteristics were measured by a Keithley 617 picoammeter. A high-intensity xenon lamp $(500 \mathrm{~W})$ was used as the UVvisible light source. The spectral response of the device was corrected for the response of the lamp-monochromator sys-

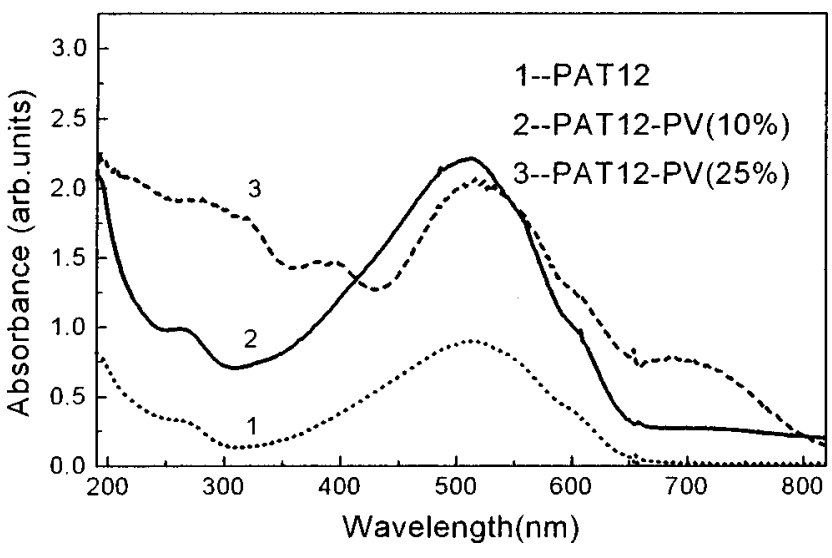

FIG. 2. The absorption spectra of PAT12 (curve 1) and PAT12-PV composite film with PV contents of $10 \%$ (curve 2) and $25 \%$ (curve 3 ).

tem by measuring the calibration spectrum with an UVenhanced Si photodiode placed in the sample position.

\section{RESULTS AND DISCUSSION}

Figure 2 indicates the change of the absorption spectra of PAT12 films containing 0, 10 and $25 \mathrm{wt} \% \mathrm{PV}$ at room temperature. As is evident from this figure, the absorption spectrum changes markedly with increasing PV content. It should be noted that the absorption peak intensity of $510 \mathrm{~nm}$ increased greatly as did the absorption baselines when the PV content was 10\%, which suggests that the absorption wavelength range of the composite film will be enlarged to the entire visible wavelength range. When the PV content is increased to $25 \%$, although the absorption peak intensity of $510 \mathrm{~nm}$ is slightly lower, some new broad and strong absorption peaks evolve at $270-320 \mathrm{~nm}, 380 \mathrm{~nm}$, and 660-800 nm, which indicate the absorption over a very broad spectral range.

Figure 3 shows the absorption spectra of the composite film before and after annealing with $25 \mathrm{wt} \% \mathrm{PV}$. Although no spectral shift of the features is apparent, the overall absorption intensity increased considerably after annealing; in particular the absorption peaks at 200-300 nm, 450-650 nm, and $650-750 \mathrm{~nm}$ are broad and strong. These phenomena

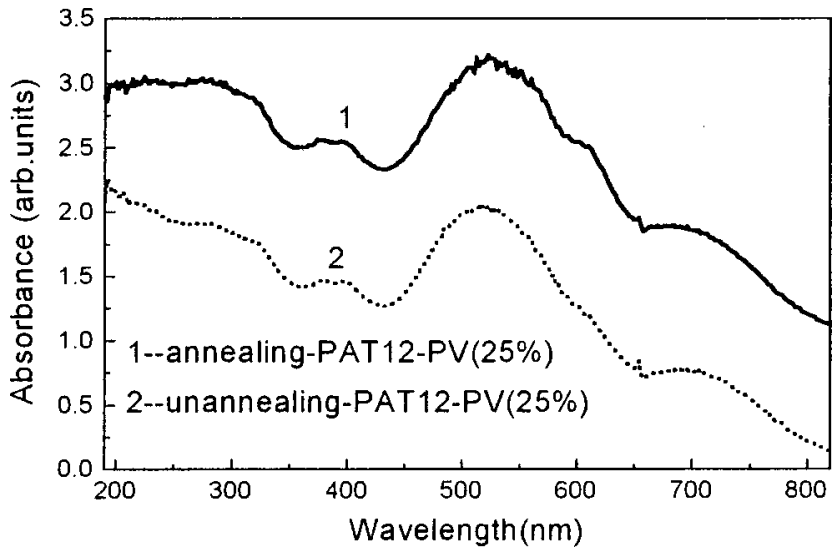

FIG. 3. The absorption spectra of PAT12-PV (25\%) composite film unannealed (curve 2) and annealed (curve 1). 


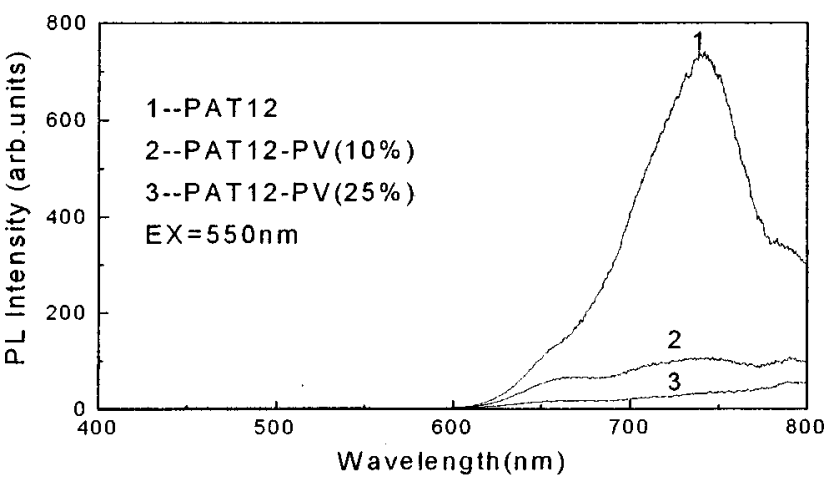

FIG. 4. Dependence of photoluminescence intensity of PAT12 (curve 1) and PAT12-PV composite film with PV contents of $10 \%$ (curve 2) and $25 \%$ (curve 3).

can be explained as follows. Among many conjugated polymers the PAT chain is very flexible. When annealed, PAT chains rearrange to reach the ordered state, which exhibits absorption in the long-wavelength range. Because of the heating, not only do the PAT chains themselves have a chance to relax and reach a more ordered state, but so does the mixture as a whole. An electron-conducting network of interconnecting PV and PAT will form in the PAT-PV composite film, which increases the absorption of the composite film over the visible light range.

The quenching of photoluminescence is also confirmed in PAT upon PV doping. As shown in Fig. 4, the photoluminescence spectra of PAT changed drastically upon PV doping; the intensity is markedly suppressed and the shape of the emission peak at $740 \mathrm{~nm}$ changes from sharp to broad and not prominent, which indicates efficient exciton dissociation in the composite. This can be explained as follows. Excitons reach the PAT/PV interface within their lifetime, where they dissociate by electron transfer from PAT to PV, resulting in PL quenching. This process also improves the characteristics of solar cells, as in the case of the conducting polymer $/ \mathrm{C}_{60}$ system. $^{8}$

UV/visible absorption and photoluminescence measurements indicate that PAT-PV composites can be used as photovoltaic materials for manufacturing photovoltaic cells.

The photovoltaic effects in the PAT-PV and PAT-PV-C ${ }_{60}$ composites were studied by fabricating doublelayer devices, ITO/PAT/PV/Al, ITO/PAT-PV/PV/Al, and ITO/PAT-PV- $\mathrm{C}_{60} / \mathrm{PV} / \mathrm{Al}$. All of these devices were annealed at $150^{\circ} \mathrm{C}$ for $1 \mathrm{~h}$. Figure 5 shows the photocurrent spectra of the devices illuminated by visible light. Spectra 1, 2 and 3 correspond to the photovoltaic devices fabricated with a single component, double components and triple components. Through comparison of the photocurrent spectra of these devices, the following specific features were revealed.

In the photocurrent spectrum of the single-composite device (ITO/PAT/PV/Al), there are rather weak responses at 328 and $643 \mathrm{~nm}$ illuminated by visible light. However, the photocurrent spectrum of the double-composite device (ITO/PAT-PV/PV/Al) reveals that the cell is able to utilize light of the entire visible spectrum. Two very strong photocurrent peaks at 400 and $636 \mathrm{~nm}$ appear in the photocur-

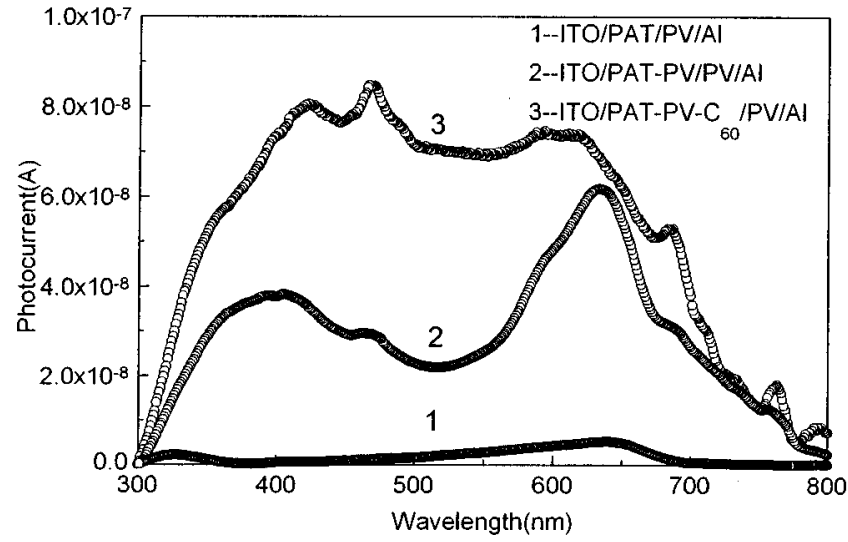

FIG. 5. The spectral dependence of photocurrent of three different composition devices. Spectra 1, 2, and 3 correspond to single-, double-, and triplecomposition devices, respectively.

rent spectrum of the double-composite device. The highest photocurrent peak is at approximately $636 \mathrm{~nm}$, which is nearly 12 times as high as that of the single-composite device. This suggests that the bulk of the cell contributes to the generation of the external photocurrent. Comparing the photocurrent spectrum in Fig. 5 and the absorption spectrum in Fig. 3, we can find that the peak of $550 \mathrm{~nm}$ in the absorption spectrum shifts to $635 \mathrm{~nm}$ in the photocurrent spectrum. The shift to long wavelength is owing to the different thicknesses of the films. Although the photocurrent spectrum of the double-composite device reveals stronger responses to the entire visible light range, some improvement is needed in the wavelength range of 400 to $600 \mathrm{~nm}$.

In order to compensate for this insufficiency, we developed the device component using triple-composite device (ITO/PAT-PV-C $60 / \mathrm{PV} / \mathrm{Al}$ ). In this way it is possible to attain the desired coverage of the broad wavelength range, and, simultaneously, ensure that the photocurrent does not decrease. The experimental results have indeed shown the desired broad spectral photoresponse. Furthermore, the photocurrent of the triple-composite device increases slightly, which indicates that efficient charge separation takes place via successive electron transfer, from PAT to $\mathrm{C}_{60}$ as well as to $\mathrm{PV}$ in the composite film and from PAT to the PV layer in the junction interface, leading to improved conversion efficiency.

The corresponding current-voltage characteristics in the dark and under illumination are shown in Fig. 6. Figures 6(a) -6 (c) correspond to the $I-V$ characteristics of the singlecomposite device, the double-composite device and the triple-composite device, respectively. All devices were irradiated with $635 \mathrm{~nm}$ light $69 \mu \mathrm{W} / \mathrm{cm}^{2}$ in intensity from the side of the ITO electrode.

The current-voltage characteristics of heterojunction devices show linear dependence of the current on the applied electric field in the dark. This indicates that a very small amount of charges is transferred through the heterostructure, resulting in a weak built-in field. However, a large increase of the photocurrent in the devices, for the forward bias, and a slight increase, for the reverse bias, are observed upon illu- 

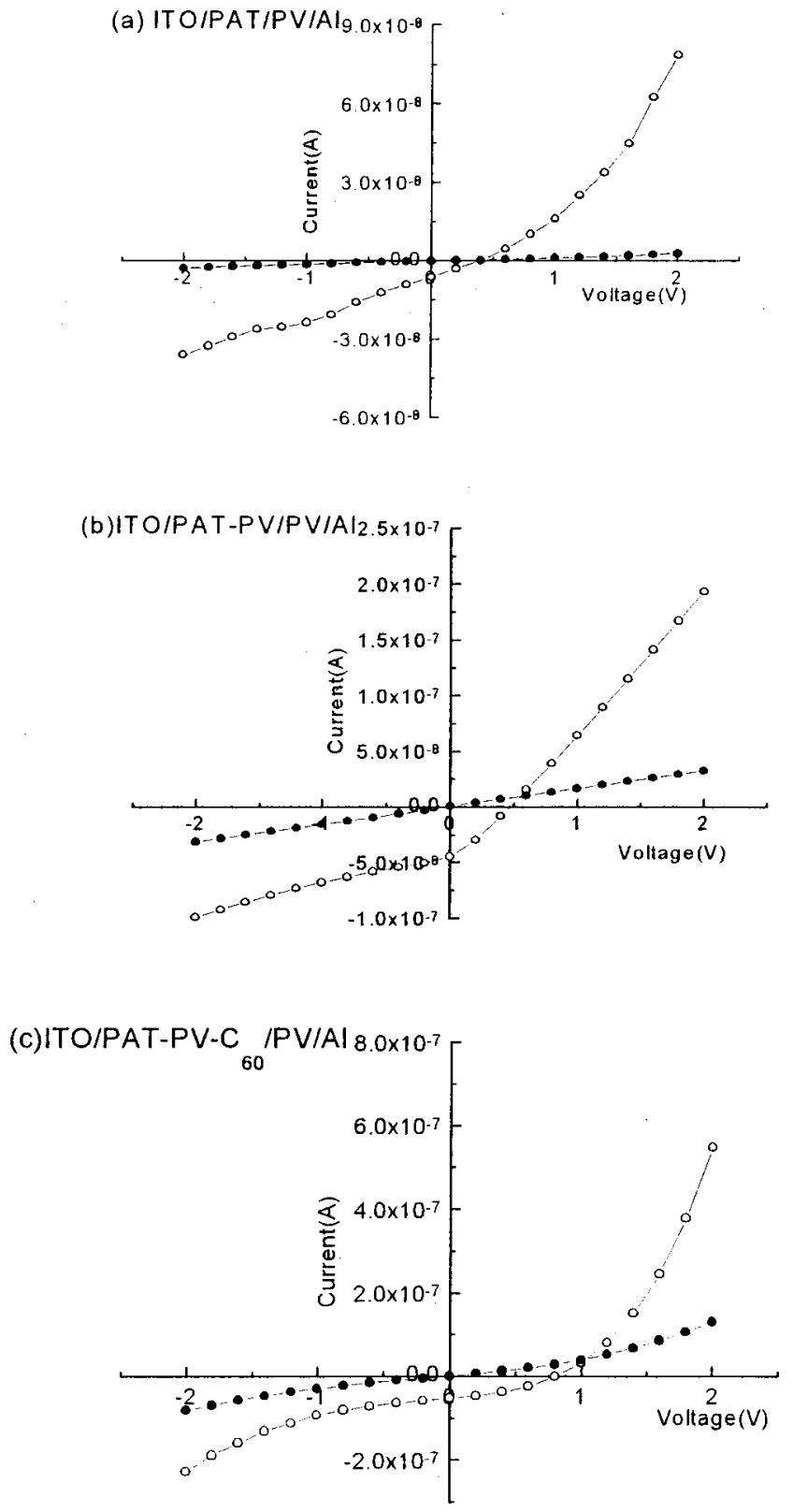

FIG. 6. The current-voltage characteristics in the dark (-) and under illumination (O). (a), (b), and (c) correspond to the $I-V$ characteristics of single-, double-, and triple-composition devices, respectively. All devices are irradiated with light of $635 \mathrm{~nm}$ and an intensity of $69 \mu \mathrm{W} / \mathrm{cm}^{2}$ from the ITO electrode.

mination with visible light. The photovoltaic characteristics of devices studied are shown in Table I.

The experimental results show that the triple-composite device exhibits a higher open-circuit voltage, short-circuit current, fill factor and conversion efficiency than do the single-composite and double-composite devices under irradiation with monochromatic light of $635 \mathrm{~nm}$. We get an increase of about one order of magnitude of the conversion efficiency between the single-composite device and the double-composite device. It results from the PV and PAT molecular rearrangement effect upon annealing which implies the storage of an internal electric field in the composite film. In this configuration, excitons must be separated more easily and the mobility of charges is improved. From the
TABLE I. Photovoltaic characteristics of devices.

\begin{tabular}{lcccl}
\hline \hline \multicolumn{1}{c}{ Device structure } & $V_{\text {oc }}(\mathrm{mV})$ & $I_{\text {sc }}\left(\mu \mathrm{A} / \mathrm{cm}^{2}\right)$ & FF & $\eta(\%)$ \\
\hline ITO/PAT/PV/Al & 380 & 0.54 & 0.227 & 0.067 \\
ITO/PAT-PV/PV/Al & 460 & 4.47 & 0.288 & 0.86 \\
ITO/PAT-PV-C 60 /PV/Al & 795 & 5.15 & 0.374 & 2.23 \\
\hline \hline
\end{tabular}

Note: Fill factor (FF) is defined as $\mathrm{FF}=I_{\max } V_{\max } / I_{\mathrm{sc}} V_{\mathrm{oc}}$. Conversion efficiency $(\eta)$ is defined as $\eta=I_{\text {sc }} \times V_{\text {oc }} \times \mathrm{FF} / P_{\text {opt }}(\lambda)$, where $P_{\text {opt }}(\lambda)$ denote the incident light flux in $\mathrm{W} \mathrm{cm}^{-2}$.

photocurrent spectrum and $I-V$ characteristic of the triplecomposite device, we can infer that upon annealing, an interpenetrating network forms in the composite of conjugated polymer with $\mathrm{PV}$ and $\mathrm{C}_{60}$, leading to a large interface area on a scale comparable with the exciton diffusion length. Thus, charge separation and charge transport are efficient in these polymer composite systems. These results give evidence that photogeneration and charge transport at the molecular level improve the photovoltaic efficiency of the polymeric semiconductor devices.

\section{SUMMARY}

The excellent photosensitivity and relatively high photovoltaic conversion efficiency obtained from photovoltaic devices fabricated using polymer-dye composite are promising. Specifically, we report on the triple-composite of PAT-PV-C 60 which shows strong and broad spectral photosensitivity and can be used in two-layer devices with PV to provide significant enhancement in efficiency. Spectral sensitization in the broad spectral range from 300 to $800 \mathrm{~nm}$, quenching of luminescence upon PV doping, and annealing effects on photoresponse and photovoltaic conversion are also reported.

${ }^{1}$ K. Granstrom, K. Petritsch, A. C. Arias, A. Lux, M. R. Andersson, and R. H. Friend, Nature (London) 395, 257 (1998).

${ }^{2}$ A. Fujii, A. Zakhidov, V. Borovkov, Y. Ohmori, and K. Yoshino, Jpn. J. Appl. Phys., Part 2 35, L1438 (1996).

${ }^{3}$ T. Kawai, T. Yamaue, K. Tada, M. Onoda, S. Jin, S. Choi, and K. Yoshino, Jpn. J. Appl. Phys., Part 2 35, L741 (1996).

${ }^{4}$ K. Yoshino, K. Tada, A. Fujii, E. M. Conwell, and A. A. Zakhidov, IEEE Trans. Electron Devices 44, 1315 (1997).

${ }^{5}$ K. Yoshino, S. Nakajima, D. H. Park, and R. Sugimoto, Jpn. J. Appl. Phys., Part 2 27, L716 (1988).

${ }^{6}$ K. Yoshino, K. Nakao, and R. Sugimoto, Jpn. J. Appl. Phys., Part 228 , L490 (1989).

${ }^{7}$ K. Tada, M. Onoda, H. Nakayama, and K. Yoshino, Synth. Met. 102, 982 (1999).

${ }^{8}$ S. Morita, A. A. Zakhidov, and K. Yoshino, Solid State Commun. 82, 249 (1992).

${ }^{9}$ C. W. Tang, Appl. Phys. Lett. 48, 183 (1986).

${ }^{10}$ D. Wohrle and D. Meissner, Adv. Mater. 3, 129 (1991).

${ }^{11}$ T. Tsutsui, T. Nakashima, T. Fujita, and S. Saito, Synth. Met. 7, 1281 (1995)

${ }^{12}$ N. Serdar Sariciftci, Curr. Opin. Solid State Mater. Sci. 4, 373 (1999).

${ }^{13}$ J. J. Dittuer, R. Lazzaroni, P. Leclere, P. Moretti, M. Granstrom, K. Petritsch, E. A. Marseglia, R. H. Friend, J. L. Bredas, H. Rost, and A. B. Holmes, Sol. Energy Mater. Sol. Cells 61, 53 (2000).

${ }^{14}$ T. Tsuzuki, Y. Shirota, J. Rostalski, and D. Meissner, Sol. Energy Mater. Sol. Cells 61, 1 (2000).

${ }^{15}$ W. Feng, A. Fujii, S. Lee, H. Wu, and K. Yoshino, Jpn. J. Appl. Phys., Part 1 39, 4978 (2000).

${ }^{16}$ K. Yoshino, S. Hayashi, and R. Sugimoto, Jpn. J. Appl. Phys., Part 223 , L899 (1984). 